\title{
Effects of Rituximab on the Development of Viral and Fungal Infections in Renal Transplant Recipients
}

\author{
Samir J. Patel, ${ }^{1}$ Jennifer M. Devos, ${ }^{1}$ Richard J. Knight, ${ }^{2}$ Kyle L. Dawson, ${ }^{1}$ Wadi N. Suki, ${ }^{3}$ \\ Juan M. Gonzalez, ${ }^{3}$ Abdul A. Abdellatif, ${ }^{4}$ and A. Osama Gaber ${ }^{2}$ \\ ${ }^{1}$ Department of Pharmacy, Houston Methodist Hospital, 6565 Fannin Street, DB1-09, Houston, TX 77030, USA \\ ${ }^{2}$ Department of Surgery, Houston Methodist Hospital, 6550 Fannin Street, SM1661, Houston, TX 77030, USA \\ ${ }^{3}$ Department of Nephrology, Houston Methodist Hospital, 6550 Fannin Street, Suite 1001, Houston, TX 77030, USA \\ ${ }^{4}$ Department of Nephrology, Baylor College of Medicine, 1709 Dryden Street, Houston, TX 77030, USA
}

Correspondence should be addressed to Samir J. Patel; spatel2@tmhs.org

Received 19 August 2013; Accepted 8 September 2013

Academic Editors: P. Ditonno and M. Veroux

Copyright (C) 2013 Samir J. Patel et al. This is an open access article distributed under the Creative Commons Attribution License, which permits unrestricted use, distribution, and reproduction in any medium, provided the original work is properly cited.

\begin{abstract}
Background. Rituximab is becoming increasingly utilized in renal transplant recipients; however, its association with infections remains unclear. Methods. We reviewed the incidence of viral and fungal infections in kidney transplant recipients treated with $(n=55)$ or without $(n=386)$ rituximab (RTX) in addition to standard immunosuppression. Results. Infections occurred in 134 (30\%) patients, with a greater proportion in RTX versus no RTX patients (47\% versus $28 \% ; P=0.005)$. Viral infections occurred in $44 \%$ and $27 \%$ of RTX and no RTX patients, respectively $(P=0.012)$. This was largely driven by the frequency of BK viremia and noncytomegalovirus/non-BK viruses in RTX patients (27\% versus $13 \%(P=0.011)$ and $15 \%$ versus $2 \%(P<0.001)$, resp.). Fungal infections also occurred more often in RTX patients (11\% versus $3 \% ; P=0.009)$. Multivariate analysis revealed deceased donor recipient (odds ratio $=2.5 ; P<0.001$ ) and rituximab exposure (odds ratio $=2.2 ; P=0.016$ ) as independent risk factors for infection. Older patients, deceased donor recipients, those on dialysis longer, and those with delayed graft function tended to be at a greater risk for infections following rituximab. Conclusions. Rituximab is associated with an increased incidence of viral and fungal infections in kidney transplantation. Additional preventative measures and/or monitoring infectious complications may be warranted in those receiving rituximab.
\end{abstract}

\section{Introduction}

The anti-CD20 monoclonal antibody rituximab (RTX) has become a more widely utilized therapy in the renal transplant population. RTX use has expanded from treatment of posttransplant lymphoproliferative disease to facilitation of ABO-incompatible transplantation, reduction of human leukocyte antigen (HLA) antibodies in highly sensitized patients, treatment of antibody mediated rejection (AMR), and treatment of recurrent and de novo renal diseases [14]. Each of these conditions represents a significant challenge in renal transplantation by which B-cell depletion with RTX may represent a promising intervention.

It is well known that, with more potent immune suppression, the risk of infectious complications after transplant increases. T-lymphocyte depleting preparations in particular have been associated with an increased risk, as opposed to nonlymphocyte depleting agents which demonstrated similar infectious risks when compared to placebo $[5,6]$. RTX has generally been considered to have an acceptable safety profile in hematological and autoimmune disorders; however, whether it can be used without an increased risk of infectious complications remains controversial in the immunosuppressed population [3, 7-14].

The purpose of the current study was to compare the incidence and type of viral and fungal infections in a consecutive group of kidney transplant patients receiving RTX in addition to standard immunosuppressive therapies to patients not exposed to RTX. Risk factors and time to develop infection are also examined. This study characterizes 
a large series of patients receiving a similar maintenance immunosuppression, a uniform prophylaxis regimen, and routine posttransplant screening for BK and cytomegalovirus (CMV) replication.

\section{Materials and Methods}

This was a retrospective, observational study of consecutive adult kidney-alone and combined kidney-pancreas recipients transplanted at a single center between January 2007 and July 2010. Patients experiencing graft loss in the first month and subjects who did not receive antibody induction therapies perioperatively were excluded. The study was approved by The Methodist Hospital Institutional Review Board.

Choice of antibody induction was determined by the center's immunologic risk-based protocol. Low-risk patients, defined as non-African American patients with panel reactive antibody (PRA) less than 20\%, received interleukin-2 receptor antagonist (IL2ra) therapy with either daclizumab (Zenapax, Roche, Nutley, NJ, USA) $2 \mathrm{mg} / \mathrm{kg}$ IV or basiliximab (Simulect, Novartis, East Hanover, NJ, USA) $20 \mathrm{mg}$ IV, on post-operative days 0 and 3; high-risk patients, defined as African Americans, patients with PRA $>20 \%$, repeat transplants, and combined kidney-pancreas recipients, received anti-thymocyte globulin [ATG; (Thymoglobulin, Genzyme, Boston, MA)] $1.5 \mathrm{mg} / \mathrm{kg} /$ dose for 3-5 doses beginning intraoperatively. Dosages were adjusted in accordance with the product's prescribing information [15]. Patients with HLA antibodies against their donor received perioperative RTX (Rituxan, Genentech, San Francisco, CA, USA) as a part of a desensitization strategy in conjunction with ATG induction. When used for desensitization, RTX was administered as a $1000 \mathrm{mg}$ dose. Patients with a historical positive cytotoxic crossmatch due to donor specific antibodies (DSA) also received IV immunoglobulin (IVIG) and therapeutic plasmapheresis in addition to RTX. Due to the long half-life and pharmacodynamic effects in patients with end stage renal disease $[16,17]$, patients receiving RTX for desensitization prior to transplantation were also included in this study. Indications for posttransplant administration of antibodies included biopsy-proven cellular rejection (Banff score > 1a) for ATG, and AMR, DSA development, and glomerulopathies for RTX.

Maintenance immunosuppression consisted of tacrolimus, mycophenolate mofetil, and prednisone. IV methylprednisolone was given intraoperatively and tapered to an oral prednisone dosage of $5-10 \mathrm{mg}$ daily by POD 60 . Mycophenolate mofetil was administered at a goal dose of 2 grams/day. Tacrolimus trough levels were maintained at 8-12 ng/mL for the first 6 months and 5-10 ng/mL thereafter.

Infection prophylaxis was administered following transplantation and treatment of rejections. This included clotrimazole four times daily for 1 month, sulfamethoxazoletrimethoprim single strength daily (or inhaled pentamidine for sulfa-allergic patients) for 6-12 months, and valcyte $450 \mathrm{mg}$ daily for 3 months in nonhigh risk CMV transplants, and 6 months in high-risk (i.e., $\mathrm{D}+/ \mathrm{R}-$ ) recipients. Pancreas recipients received 1 month of fluconazole $200 \mathrm{mg}$ daily instead of clotrimazole following transplantation. Routine CMV and BK polymerase chain reaction (PCR) screening was employed at 1, 3, 6, 9, and 12 months and then every 6 months after transplant and whenever clinically warranted.

The primary endpoint of the study was the incidence of viral and fungal infections among RTX-treated patients (RTX group) compared to patients not exposed to RTX (no RTX). Only infectious complications occurring after the antibody exposure were included in the analysis. The following infections were included in the analysis: (1) CMV infection: defined as a detectable viral load in plasma ( $>300$ copies $/ \mathrm{mL}$ ) measured by PCR and/or viral inclusions on histopathologic specimens, in combination with compatible symptoms; (2) BK virus infection: defined as detectable BK viral DNA in plasma (>750 copies $/ \mathrm{mL}$ ) in two consecutive measurements. All BK-viremic patients underwent protocol biopsy for presence of nephropathy; (3) "other" viral infections: defined as viral infections other than $\mathrm{CMV}$ or $\mathrm{BK}$ diagnosed by serologic testing, quantitative viral load measurements, and histopathologic assessment in conjunction with attributable signs and symptoms; and (4) fungal infections: defined as any positive fungal culture or staining of biopsy material, excluding oral candidiasis. Secondary endpoints included the incidence and types of specific infections, time to infection, and subgroup analyses based on IVIG administration and retransplantation status.

Continuous variables including recipient age, peak PRA, cumulative ATG dosages, and total number of infections per patient were compared using Student's $t$-tests. Gender, race, presence of diabetes, donor type, history of prior transplant, high-risk CMV status, immunosuppressive type, and incidence of infections were compared using chi-squared or Fisher's exact test. Due to the difference in sample sizes, categorical variables listed in Tables Tables 1-3 are shown as $\%(n)$. A univariate analysis of risk factors for infection was performed, and those factors with a $P$ value of less than 0.2 were included in a nominal logistic multivariate analysis. Statistical analysis was performed using JMP version 7.0 (SAS Institute, Cary, NC, USA).

\section{Results}

A total of 453 kidney-only or kidney-pancreas transplants were performed during the study period. Seven patients were excluded for not receiving the antibody therapies at the time of transplantation, and 5 were excluded for graft loss within the first month. Characteristics of the remaining 441 patients included a mean age of $48 \pm 14$ years, $59 \%$ male, $61 \%$ deceased donor, and $43 \%$ Caucasian. Diabetes (30\%) and hypertension (23\%) were the leading causes of end stage renal disease. Fiftyfive patients comprised the RTX group. Median followup for the entire cohort was 31 months (range from 3 to 56), with no difference in mean followup between groups. Major differences between the groups were driven by our desensitization protocol in which there was a predominance of Caucasian living donor transplant recipients, highly sensitized patients, and patients receiving a repeat transplant (Table 1 ). 
TABLE 1: Demographics and transplant characteristics.

\begin{tabular}{|c|c|c|c|}
\hline Variable & $\begin{array}{c}\text { RTX } \\
n=55\end{array}$ & $\begin{array}{c}\text { No RTX } \\
n=386\end{array}$ & $P$ value \\
\hline Age, years & $47 \pm 14$ & $49 \pm 14$ & 0.42 \\
\hline \multicolumn{4}{|l|}{ Race } \\
\hline Caucasian, $\%(n)$ & $65(36)$ & $40(155)$ & $<0.001$ \\
\hline African American, $\%(n)$ & $18(10)$ & $27(104)$ & 0.15 \\
\hline Male gender, \% ( $n)$ & $42(23)$ & $61(235)$ & 0.008 \\
\hline Diabetes history, \% ( $n$ ) & $24(13)$ & $36(137)$ & 0.08 \\
\hline Concurrent viral disease ${ }^{\mathrm{a}} \%(n)$ & $4(2)$ & $6(24)$ & 0.42 \\
\hline Peak PRA, \% & $53 \pm 41$ & $20 \pm 31$ & $<0.001$ \\
\hline Deceased donor, $\%(n)$ & $45(25)$ & $38(145)$ & 0.26 \\
\hline Retransplant, \% (n) & $24(13)$ & $7(27)$ & $<0.001$ \\
\hline Pretransplant dialysis, years & $3.2 \pm 4$ & $2.7 \pm 3$ & 0.27 \\
\hline Kidney-pancreas recipient, $\%(n)$ & $9(5)$ & $7(26)$ & 0.54 \\
\hline CMV donor+/recipient- serostatus & $22(12)$ & $17(67)$ & 0.43 \\
\hline Delayed graft function & $9(5)$ & $9(36)$ & 0.97 \\
\hline
\end{tabular}

Abbreviations-PRA: panel reactive antibodies; CMV: cytomegalovirus.

${ }^{\text {a }}$ Concurrent viral disease includes history of hepatitis B, hepatitis C, or human Immunodeficiency virus.

TABLE 2: Baseline and posttransplant immunosuppression.

\begin{tabular}{|c|c|c|c|}
\hline Variable & $\begin{array}{c}\text { RTX } \\
n=55\end{array}$ & $\begin{array}{l}\text { No RTX } \\
n=386\end{array}$ & $P$ value \\
\hline IL2ra induction, \% $(n)$ & $18(10)$ & $40(156)$ & $<0.001$ \\
\hline Perioperative ATG, \% $(n)$ & $84(46)$ & $63(242)$ & 0.001 \\
\hline Perioperative IVIG, $\%(n)$ & $33(18)$ & $2(5)$ & $<0.001$ \\
\hline Tacrolimus at discharge, $\%(n)$ & $100(55)$ & $100(386)$ & \\
\hline Mycophenolate at discharge, $\%(n)$ & $100(55)$ & $100(386)$ & \\
\hline Prednisone withdrawal, \% $(n)$ & $5(3)$ & $13(51)$ & 0.07 \\
\hline Acute rejection, \% (n) & $51(28)$ & $15(56)$ & $<0.001$ \\
\hline Antibody-mediated rejection, $\%(n)$ & $23(12)$ & $3(11)$ & $<0.001$ \\
\hline ATG for rejection, $\%(n)$ & $29(16)$ & $7(28)$ & $<0.001$ \\
\hline Total ATG exposure, $(\mathrm{mg} / \mathrm{kg})$ & $8.5 \pm 6.5$ & $5.7 \pm 2.4$ & $<0.001$ \\
\hline
\end{tabular}

Abbreviations-IL2ra: interleukin-2 receptor antagonist; ATG: antithymocyte globulin; IVIG: intravenous immune globulin.

${ }^{a}$ Concurrent viral disease includes history of hepatitis B, hepatitis C, or human Immunodeficiency virus.

A significantly greater percentage of RTX patients received perioperative IVIG and/or ATG as a result of their higher immunologic profile (Table 1). There were no differences in tacrolimus trough levels, mycophenolate dosages, or prednisone dosages throughout the study period (data not shown). Rejection episodes, including those requiring treatment with ATG, occurred more frequently in the RTX group, and RTX patients received significantly more ATG post-transplant (Table 2).

A total of 64 RTX doses were administered to the 55 RTX patients. Indications included desensitization of a living donor recipient $(n=18)$, rejection $(n=16)$, desensitization of a deceased donor recipient $(n=15)$, DSA formation $(n=8)$, and recurrence of a glomerulopathy $(n=7)$. Nine patients received a second dose of RTX, and none received more than two. Recurrent diseases for which RTX was administered included focal segmental glomerulosclerosis $(n=3)$, membranoproliferative glomerulonephritis $(n=$ 2 ), Wegener's granulomatosis $(n=1)$, and membranous nephropathy $(n=1)$. RTX was administered at a median dose of $1000 \mathrm{mg}$ (range from 200 to $1000 \mathrm{mg}$ ) or $550 \mathrm{mg} / \mathrm{m}^{2}$ per dose based on the average body surface area of the cohort. Depending on indication, some RTX dosages were administered concomitantly with additional therapies including ATG (58\%), IVIG (47\%), and plasmapheresis (23\%). All patients receiving plasmapheresis were also administered IVIG. In addition, 2 RTX-treated patients received bortezomib as part of an antirejection regimen. The median and mean times from transplantation to first dose were 2 days (range from -265 to 827$)$ and $99 \pm 214$ days, respectively.

At least one infection occurred in $134(30 \%)$ of the 441 patients, at a median of 5.1 (range 0.4 to 28.5) months after 


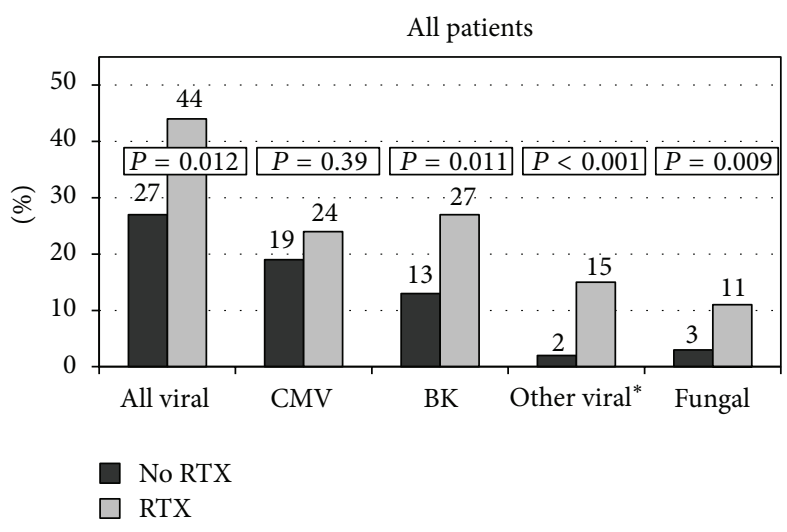

(a)

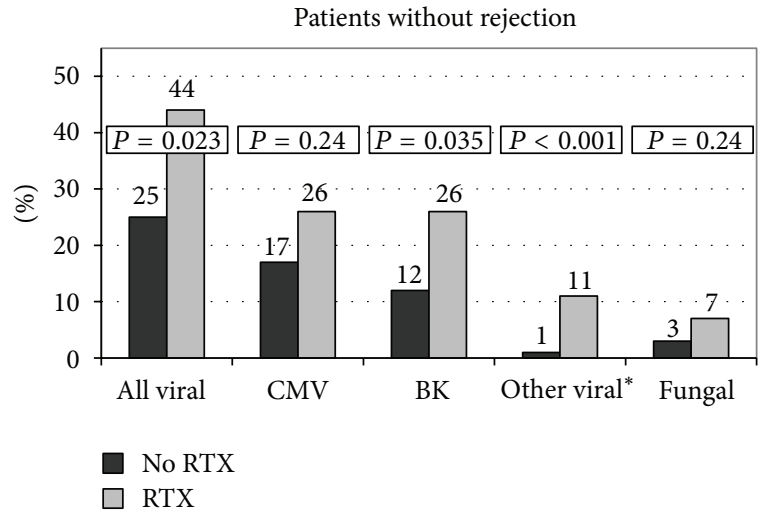

(b)

FIGURE 1: (a) Incidence of viral and fungal infections according to rituximab exposure in the entire cohort. (b) Incidence of viral and fungal infections, excluding patients treated for acute rejection. ${ }^{*}$ represents all viral infections except for cytomegalovirus and BK.

antibody exposure. The combined incidence of viral and fungal infections was significantly higher in the RTX group than in the no RTX group (47\% versus $28 \% ; P=0.005$ ). Viral infections occurred more frequently in the RTX group compared to the no RTX group ( $44 \%$ versus $27 \%$; $P=0.012$ ), owing primarily to a higher rate of $\mathrm{BK}$ virus and other non$\mathrm{CMV} /$ non-BK viral infections (Figure 1(a)). Similarly, fungal infections occurred more frequently in the RTX group (11\% versus $3 \% ; P=0.008)$. To determine the rate of infections in patients who did not require additional antirejection therapies, we reviewed the incidence of infections between groups after excluding all patients with rejection (Figure 1(b)). This resulted in findings similar to those seen in the entire cohort.

Specific types of viral and fungal infections by group are shown in Table 3 . As previously mentioned, the rate of overall viral infections seen in the RTX group was largely driven by the significantly higher rate of BK viremia and non-CMV/non-BK viruses as compared to the no RTX group (27\% versus $13 \%$ and $15 \%$ versus $2 \%$, resp.; $P<0.01$ ). BK nephropathy was diagnosed in $15 \%$ of RTX-treated patients compared $4 \%$ in the no RTX group $(P<0.001)$. There was no difference in the rate of CMV between groups. As shown in Figure 2, most cases of CMV and BK in RTXtreated patients occurred in the first 6 months after antibody exposure, whereas a number of "other" viral and fungal infections occurred after 6 months.

The most common non-CMV/non-BK viral infection was varicella zoster infection, which occurred in 4 RTX patients and 1 no RTX patient. Three RTX patients with viral infections died. These included one case of EBV-associated PTLD (occurring after RTX administration) in a living donor recipient with DSAs, a patient with respiratory syncytial virus (RSV) after receiving RTX for a rejection episode following a second transplant, and a third recipient who developed JC virus-associated progressive multifocal leukoencephalopathy (PML), approximately 30 months after receiving two doses of RTX for a severe renal and pancreas allograft rejection.

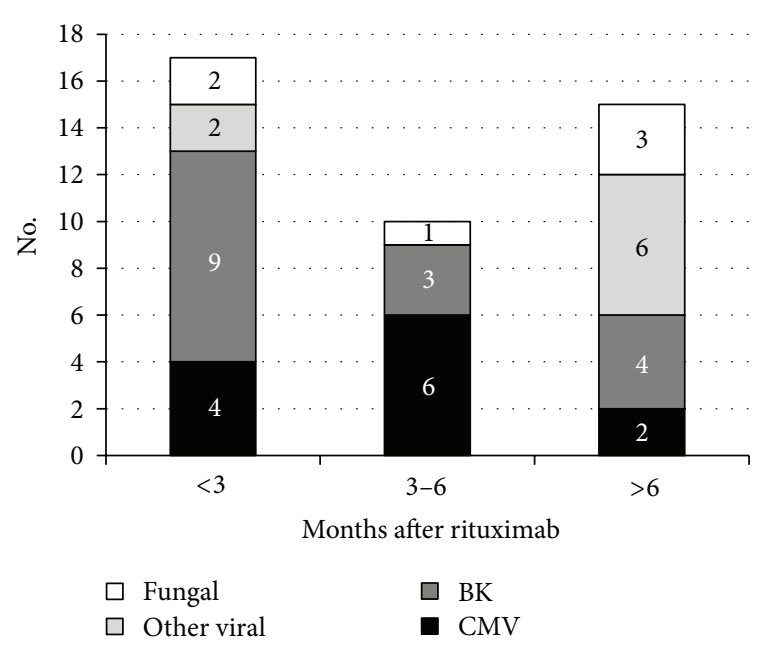

FIgURE 2: Timeline of viral and fungal infections after rituximab. Other viral infections: $<3$ months: RSV (1) and EBV (1); >6 months: varicella zoster (4), influenza H1N1 (1), and JC Virus (1). Fungal infections: $<3$ months: candida glabrata urinary tract infection (1), and disseminated tinea corpis (1); 3-6 months: candida esophagitis; $>6$ months (1): candida esophagitis, candidemia (1), nonaspergillus mould pneumonia (1), candidal brain abscess (1).

Fungal infections were more frequent in the RTX group. Of note, several severe fungal infections occurred in the no RTX group, including cases of pulmonary aspergillus (4), cryptococcal meningitis (3), mucormycosis (1), gastrointestinal invasive candidiasis (1), and candidal brain abscess (1). One patient with HCV had concurrent aspergillus and cryptococcal infections. Each patient with aspergillus and/or cryptococcal infection was treated successfully and is alive to date; however, deaths ultimately occurred in the patients with Mucor (patient with HCV, diabetes, and ATG induction), invasive candidiasis (previous liver transplant recipient with small bowel infarct shortly after transplant), and the candidal 
TABLE 3: Infectious complications.

\begin{tabular}{|c|c|c|c|}
\hline Infection & $\begin{array}{c}\text { RTX } \\
n=55\end{array}$ & $\begin{array}{l}\text { No RTX } \\
n=386\end{array}$ & $P$ value \\
\hline Viral, \% (n) & $44(24)$ & $27(103)$ & 0.012 \\
\hline CMV infection, \% (n) & $20(11)$ & $19(72)$ & 0.81 \\
\hline BK viremia, \% $(n)$ & $27(15)$ & $13(51)$ & 0.011 \\
\hline BK nephropathy, \% (n) & $15(8)$ & $4(14)$ & 0.003 \\
\hline Other viral, \% (n) & $15(8)$ & $2(6)$ & $<0.001$ \\
\hline Ebstein Barr virus, $n$ & 1 & 0 & \\
\hline Human papilloma virus, $n$ & 0 & 1 & \\
\hline Influenza A, $n$ & 0 & 2 & \\
\hline Influenza H1N1, $n$ & 1 & 2 & \\
\hline JCV-associated PML, $n$ & 1 & 0 & \\
\hline Respiratory syncytial virus, $n$ & 1 & 0 & \\
\hline Varicella zoster, $n$ & 4 & 1 & \\
\hline Number of viral infections/patient & 0.6 & 0.3 & 0.005 \\
\hline Fungal, \% (n) & $11(6)$ & $3(10)$ & 0.009 \\
\hline Aspergillosis, pulmonary, $n$ & 0 & 4 & \\
\hline Candida infections, $n$ & 4 & 3 & \\
\hline Cryptococcal meningoencephalitis, $n$ & 0 & 3 & \\
\hline Nonaspergillus mould, pulmonary, $n$ & 1 & 0 & \\
\hline Mucormycosis, pulmonary, $n$ & 0 & 1 & \\
\hline Tinea corpis, disseminated, $n$ & 1 & 0 & \\
\hline Number of fungal infections/patient & 0.11 & 0.03 & 0.006 \\
\hline
\end{tabular}

Abbreviations-CMV: cytomegalovirus; JCV: JC virus; PML: progressive multifocal leukoencephalopathy.

brain abscess (62-year-old male with diabetes and ATG induction). Among RTX-treated patients, one episode of candidemia occurred, which was successfully treated, and one case of nonaspergillus mould pneumonia occurred in a previous kidney-pancreas recipient who eventually succumbed to respiratory failure. This patient had received ATG and RTX for antibody-mediated rejection one year prior.

Univariate and multivariate analyses of factors associated with the development of infection posttransplant are shown in Table 4. Recipients of deceased donor transplants and RTX-treated patients were independently associated with development of infections, with odds ratios of 2.5 and 2.2, respectively. Among the 55 RTX-treated patients, a univariate analysis of risk factors for infection was performed (not shown). No significant risk factors for infection among rituximab treated patients were identified, although several factors approached significance. Patients developing infection tended to be older (51 versus $44 \mathrm{yrs} ; P=0.06$ ), deceased donor recipients (57\% versus $36 \%$ for living donors recipients; $P=0.12$ ), on dialysis for a greater duration pretransplant (4.3 versus 2.2 years; $P=0.07$ ), and more likely to have delayed graft function ( $80 \%$ versus $43 \%$ in those with immediate function $P=0.10)$.

In considering a potential immune-protective effect of IVIG, we compared the incidence of infections in RTX patients according to concomitant IVIG use. There was no difference in the rate of infection among the 26 patients receiving IVIG to those not receiving IVIG (50\% versus $45 \%$;
$P=0.72$ ). Additionally, there may be a concern that the patients receiving RTX for retransplantation may be at greater risk for infection due to long-standing immunosuppression. We therefore compared the incidence of infections in patients undergoing retransplant without exposure to RTX $(n=27)$ to patients receiving RTX with a primary transplant $(n=42)$. Infections occurred in $26 \%$ of the retransplanted patients without RTX versus $49 \%$ of patients receiving RTX with a primary transplant $(P=0.06)$.

\section{Discussion}

Over the past decade, RTX use has expanded in renal transplantation, particularly in the settings of desensitization, antibody-mediated rejection, and treatment of glomerulopathies. The safety profile of RTX in this population remains unclear, however, with conflicting data on its association with infectious complications [3,7-14]. This study of 55 kidney transplant patients treated with RTX for its common peri- and posttransplant indications favors an association an increased risk of infections with RTX.

Through routine posttransplant BK screening and protocol biopsies, we observed a significantly greater incidence of both BK viremia and nephropathy among RTX-treated patients. Previously, Kamar et al. noted that BK virus was the most common virus detected in RTX-treated patients [8], and Habicht et al. found a nonsignificantly higher 
TABLE 4: Risk factors for viral or fungal infection.

\begin{tabular}{|c|c|c|c|c|c|}
\hline & $\%$ Infection & \% Infection (Comparator) & $\begin{array}{c}\text { Univariate } \\
P \text { value }\end{array}$ & Odds ratio & $\begin{array}{c}\text { Multivariate } \\
P \text { value }\end{array}$ \\
\hline Age $\geq 60$ years & 34 & $29(<60$ years $)$ & 0.39 & & \\
\hline Caucasian & 30 & 30 (non-Caucasian) & 0.98 & & \\
\hline Male gender & 29 & 32 (female) & 0.47 & & \\
\hline Diabetes history & 35 & 27 (no diabetes) & 0.08 & 1.2 & 0.47 \\
\hline History chronic virus & 23 & 31 (no virus) & 0.39 & & \\
\hline Deceased donor & 38 & 18 (living donor) & $<0.001$ & 2.5 & $<0.001$ \\
\hline Retransplant & 30 & 30 (first transplant) & 0.98 & & \\
\hline Kidney-pancreas recipient & 58 & 28 (kidney only) & $<0.001$ & 1.7 & 0.22 \\
\hline Perioperative ATG & 32 & 27 (no ATG) & 0.33 & & \\
\hline Steroids at discharge & 30 & 30 (steroid withdrawal) & 0.90 & & \\
\hline Delayed graft function & 34 & 30 (initial function) & 0.54 & & \\
\hline Acute rejection & 43 & 27 (no rejection) & 0.007 & 1.5 & 0.12 \\
\hline ATG for rejection & 34 & 30 (no ATG) & 0.58 & & \\
\hline Any ATG exposure & 32 & 27 (no ATG exposure) & 0.31 & & \\
\hline Any RTX exposure & 47 & 28 (no RTX exposure) & 0.005 & 2.2 & 0.016 \\
\hline
\end{tabular}

Abbreviations-ATG: antithymocyte globulin; RTX: rituximab.

rate of $\mathrm{BK}$ in $\mathrm{ABO}$-incompatible recipients administered RTX [11]. The rates of BK viremia and nephropathy in our cohort are congruent with rates previously reported in the renal transplant population [18-21]. However, viremia and nephropathy occurred 2-3x more often in the RTX cohort. Similarly, an increased rate of "other" viral infections (nonCMV and non-BK related) was also noted. Varicella zoster infection occurred in 4 of 55 RTX patients compared to 1 in 386 no RTX patients. Zoster infections have been reported in other series of RTX-treated patients [11] and were even fatal in some cases $[22,23]$. While none of our zoster cases suffered such fate, 3 deaths occurred among RTX patients owing to EBV-associated PTLD, RSV, and JCV-associated PML. Interestingly, we found no difference in the rate of $\mathrm{CMV}$ between groups. This may be due to the routine use of valganciclovir prophylaxis following acute rejection treatments.

A significantly higher incidence of fungal infections was noted in the RTX group, a similar finding albeit at a lower rate than Kamar's report (17\%) [8]. In contrast to their results, however, the severity of fungal infections tended to be less in our RTX patients. All severe fungal infections, including cases of aspergillosis or cryptococcal meningitis, occurred in the no RTX group. This may have been due to the baseline characteristics of these patients, with the use of antithymocyte globulin, diabetes history, and chronic hepatitis $\mathrm{C}$ infection being common amongst these cases. Each case of aspergillus and cryptococcal meningitis was successfully treated.

Finally, a surprising observation in this study was the timing of infection, with a number of infections developing late (beyond 6 months) after RTX exposure. This data indicates that perhaps prophylaxis and/or close monitoring for opportunistic infections should continue for an extended period after RTX administration.

Several possible mechanisms exist for our findings. RTX may induce hypogammaglobulinemia [24, 25] which has been associated with infections in solid organ transplant populations [26]. While IgG monitoring was not routinely performed in our cohorts it is plausible that depletion of BK or other viral-specific IgG, may have occurred. Previous data demonstrated that low BK virus IgG levels correlated with a higher degree of $\mathrm{BK}$ replication [27]. BK and other non-CMV viral infections seen in our cohort either lacked prophylactic strategies (i.e., JC virus, RSV) or occurred late (>6 months) after RTX exposure, making prevention and/or monitoring for these infections difficult. RTX may have longterm effects on B-cell depletion, especially in the setting of renal dysfunction [16, 17]. This may account for some of the late onset infections seen in our cohort. Furthermore, as $\mathrm{B}$-cells are known antigen-presenting cells, depletion of Bcells may hinder virus-specific T-cell responses and immune responses to fungal pathogens.

The role of concomitant immunosuppressive therapies should be appreciated. While we observed no differences in maintenance immunosuppressive drug levels or dosages, we did observe a higher cumulative amount of ATG given to the RTX-treated patients. To eliminate the influence of augmented immunosuppression with rejection therapies, we evaluated the incidence of viral and fungal infections in patients without any rejections. The results were similar to the cohort as a whole, suggesting that even when used without additional augmented immunosuppression, RTX may still promote the development of infections.

This study has several limitations, most of which relate to its retrospective nature. Without the use of a randomized, prospective design, it is difficult to clearly ascertain the risk of 
one antibody agent over another. As mentioned previously, IgG levels were not checked, and therefore the contribution of hypogammaglobulinemia could not be assessed. In our population, we did not observe a "protective effect" of IVIG, perhaps owing to the prolonged hypogammaglobulinemic effects observed with RTX [24, 25]. Finally, our sample size was not large enough to determine statistically significant risks for infection among the RTX-treated cohort, although trends were seen in those who were older, on dialysis longer, deceased donor transplants, and those with DGF.

In conclusion, we found a significantly higher rate of viral and fungal infections in patients treated with RTX. Most infections observed were those for which established prophylactic strategies do not exist, and some may occur late after rituximab exposure. Close monitoring for infections and expanded use of prophylactic strategies should be considered in patients receiving RTX.

\section{Abbreviations}

AMR: Antibody mediated rejection

ATG: Antithymocyte globulin

CMV: Cytomegalovirus

DSA: Donor specific antibodies

HLA: Human leukocyte antigen

IL2ra: Interleukin-2 receptor antagonist

IVIG: Intravenous immunoglobulin

PRA: Panel reactive antibody

RTX: Rituximab.

\section{Conflicts of Interests}

The authors declare no conflict of interests.

\section{Acknowledgments}

The authors would like to thank the organ transplant coordinators and clinical research coordinators at the Houston Methodist J. C. Walter Jr Transplant Center in Houston, TX, USA for their maintenance of transplant center data and assistance in attaining clinical information.

\section{References}

[1] M. D. Pescovitz, "Rituximab, an anti-CD20 monoclonal antibody: history and mechanism of action," The American Journal of Transplantation, vol. 6, no. 5, part 1, pp. 859-866, 2006.

[2] Y. T. Becker, M. Samaniego-Picota, and H. W. Sollinger, "The emerging role of rituximab in organ transplantation," Transplant International, vol. 19, no. 8, pp. 621-628, 2006.

[3] A. A. Vo, M. Lukovsky, M. Toyoda et al., "Rituximab and intravenous immune globulin for desensitization during renal transplantation," The New England Journal of Medicine, vol. 359, no. 3, pp. 242-251, 2008.

[4] H. Genberg, G. Kumlien, L. Wennberg, U. Berg, and G. Tydén, "ABO-incompatible kidney transplantation using antigenspecific immunoadsorption and rituximab: a 3-year follow-up," Transplantation, vol. 85, no. 12, pp. 1745-1754, 2008.
[5] G. L. Bumgardner, I. Hardie, R. W. G. Johnson et al., "Results of 3-year phase III clinical trials with daclizumab prophylaxis for prevention of acute rejection after renal transplantation," Transplantation, vol. 72, no. 5, pp. 839-845, 2001.

[6] B. Nashan, R. Moore, P. Amlot, A.-G. Schmidt, K. Abeywickrama, and J.-P. Soulillou, "Randomised trial of basiliximab versus placebo for control of acute cellular rejection in renal allograft recipients," The Lancet, vol. 350, no. 9086, pp. 11931198, 1997.

[7] S. A. Grim, T. Pham, J. Thielke et al., "Infectious complications associated with the use of rituximab for ABO-incompatible and positive cross-match renal transplant recipients," Clinical Transplantation, vol. 21, no. 5, pp. 628-632, 2007.

[8] N. Kamar, O. Milioto, B. Puissant-Lubrano et al., "Incidence and predictive factors for infectious disease after rituximab therapy in kidney-transplant patients," The American Journal of Transplantation, vol. 10, no. 1, pp. 89-98, 2010.

[9] A. S. Munoz, A. A. Rioveros, C. B. Cabanayan-Casasola, R. A. Danguilan, and E. T. Ona, "Rituximab in highly sensitized kidney transplant recipients," Transplantation Proceedings, vol. 40, no. 7, pp. 2218-2221, 2008.

[10] Z. Kaposztas, H. Podder, S. Mauiyyedi et al., "Impact of rituximab therapy for treatment of acute humoral rejection," Clinical Transplantation, vol. 23, no. 1, pp. 63-73, 2009.

[11] A. Habicht, V. Bröker, C. Blume et al., "Increase of infectious complications in $\mathrm{ABO}$-incompatible kidney transplant recipients-a single centre experience," Nephrology Dialysis Transplantation, vol. 26, no. 12, pp. 4124-4131, 2011.

[12] C. H. Baek, W. S. Yang, K. S. Park, D. J. Han, J. B. Park, and S. K. Park, "Infectious risks and optimal strength of maintenance immunosuppressants in rituximab-treated kidney transplantation," Nephron Extra, vol. 2, no. 1, pp. 66-75, 2012.

[13] G. Tydén, H. Genberg, J. Tollemar et al., "A randomized, doubleblind, placebo-controlled, study of single-dose rituximab as induction in renal transplantation," Transplantation, vol. 87, no. 9, pp. 1325-1329, 2009.

[14] J. Kahwaji, A. Sinha, M. Toyoda et al., "Infectious complications in kidney-transplant recipients desensitized with rituximab and intravenous immunoglobulin," Clinical Journal of the American Society of Nephrology, vol. 6, no. 12, pp. 2894-2900, 2011.

[15] Thymoglobulin (Anti-thymocyte globulin rabbit), Package Insert, Genzyme, Cambridge, Mass, USA.

[16] H. Genberg, A. Hansson, A. Wernerson, L. Wennberg, and G. Tydén, "Pharmacodynamics of rituximab in kidney transplantation," Transplantation, vol. 84, supplement 12, pp. S33-S36, 2007.

[17] C. A. Vieira, A. Agarwal, B. K. Book et al., "Rituximab for reduction of anti-HLA antibodies in patients awaiting renal transplantation: 1. Safety, pharmacodynamics, and pharmacokinetics1," Transplantation, vol. 77, no. 4, pp. 542-548, 2004.

[18] I. A. Memon, B. A. Parikh, M. Gaudreault-Keener, R. Skelton, G. A. Storch, and D. C. Brennan, "Progression from sustained $\mathrm{BK}$ viruria to sustained $\mathrm{BK}$ viremia with immunosuppression reduction is not associated with changes in the noncoding control region of the BK virus genome," Transplantation, vol. 2012, Article ID 761283, 7 pages, 2012.

[19] H. H. Hirsch, D. C. Brennan, C. B. Drachenberg et al., "Polyomavirus-associated nephropathy in renal transplantation: interdisciplinary analyses and recommendations," Transplantation, vol. 79, no. 10, pp. 1277-1286, 2005.

[20] D. C. Brennan, I. Agha, and D. L. Bohl, "Incidence of BK with tacrolimus versus cyclosporine and impact of preemptive 
immunosuppression reduction," The American Journal of Transplantation, vol. 5, no. 3, pp. 582-594, 2005.

[21] E. Ramos, C. B. Drachenberg, R. Wali, and H. H. Hirsch, "The decade of polyomavirus BK-associated nephropathy: state of affairs," Transplantation, vol. 87, no. 5, pp. 621-630, 2009.

[22] A. Bermúdez, F. Marco, E. Conde, E. Mazo, M. Recio, and A. Zubizarreta, "Fatal visceral varicella-zoster infection following rituximab and chemotherapy treatment in a patient with follicular lymphoma," Haematologica, vol. 85, no. 8, pp. 894-895, 2000.

[23] L. M. Mcilwaine, E. J. Fitzsimons, and R. L. Soutar, "Inappropriate antidiuretic hormone secretion, abdominal pain and disseminated varicella-zoster virus infection: an unusual and fatal triad in a patient 13 months post Rituximab and autologous stem cell transplantation," Clinical and Laboratory Haematology, vol. 23, no. 4, pp. 253-254, 2001.

[24] E. Irie, Y. Shirota, C. Suzuki et al., "Severe hypogammaglobulinemia persisting for 6 years after treatment with rituximab combined chemotherapy due to arrest of B lymphocyte differentiation together with alteration of T lymphocyte homeostasis," International Journal of Hematology, vol. 91, no. 3, pp. 501-508, 2010.

[25] N. Cooper, E. G. Davies, and A. J. Thrasher, "Repeated courses of rituximab for autoimmune cytopenias may precipitate profound hypogammaglobulinaemia requiring replacement intravenous immunoglobulin," British Journal of Haematology, vol. 146, no. 1, pp. 120-122, 2009.

[26] S. Mawhorter and M. H. Yamani, "Hypogammaglobulinemia and infection risk in solid organ transplant recipients," Current Opinion in Organ Transplantation, vol. 13, no. 6, pp. 581-585, 2008.

[27] S. Hariharan, E. P. Cohen, B. Vasudev et al., "BK virus-specific antibodies and BKV DNA in renal transplant recipients with BKV nephritis," The American Journal of Transplantation, vol. 5, no. 11, pp. 2719-2724, 2005. 


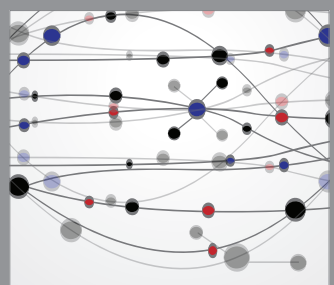

The Scientific World Journal
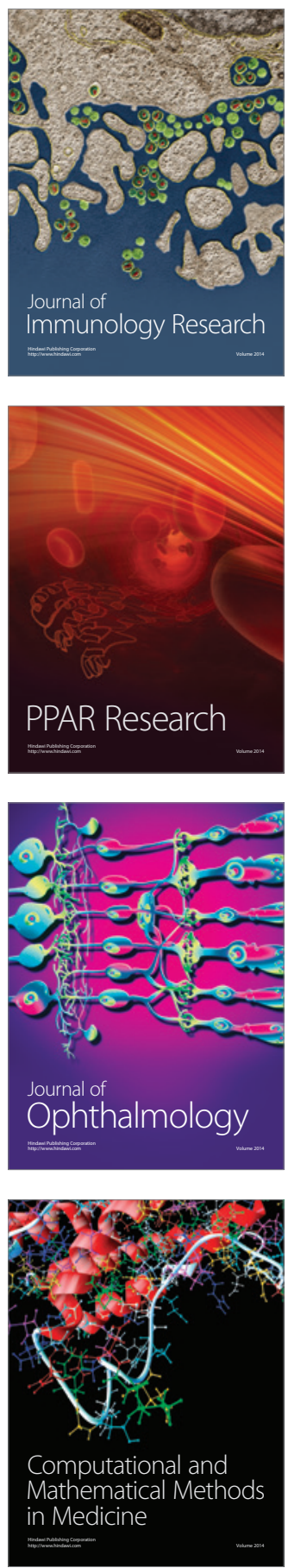

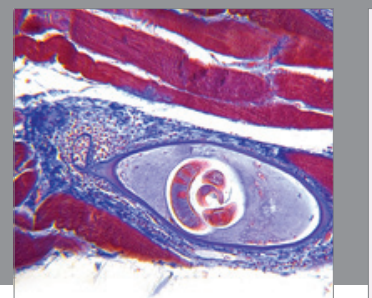

Gastroenterology

Research and Practice
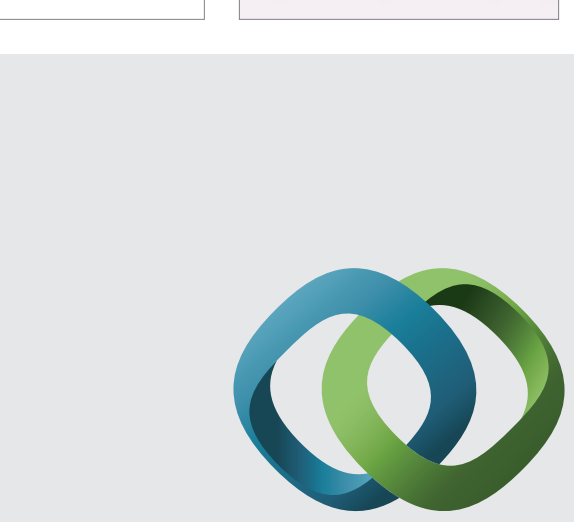

\section{Hindawi}

Submit your manuscripts at

http://www.hindawi.com
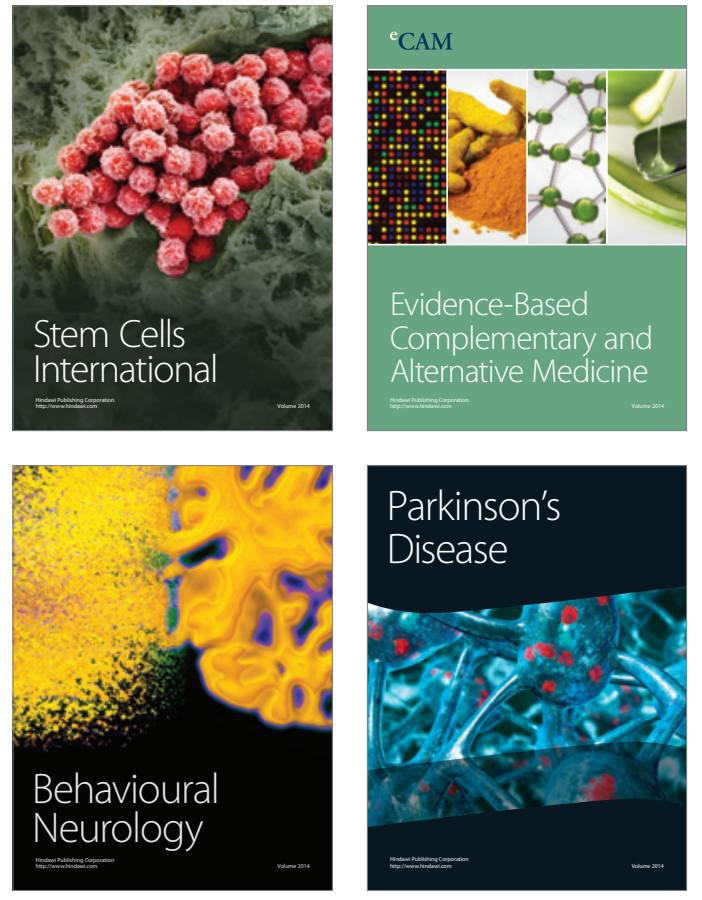
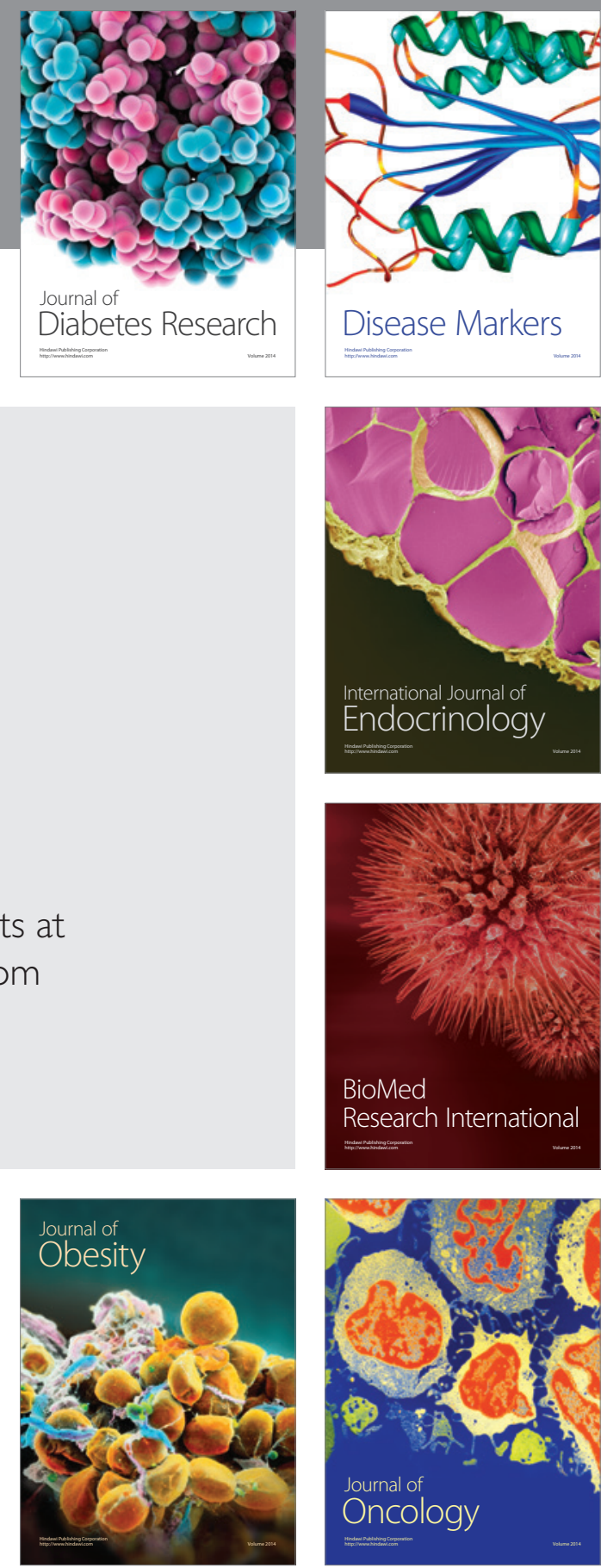

Disease Markers
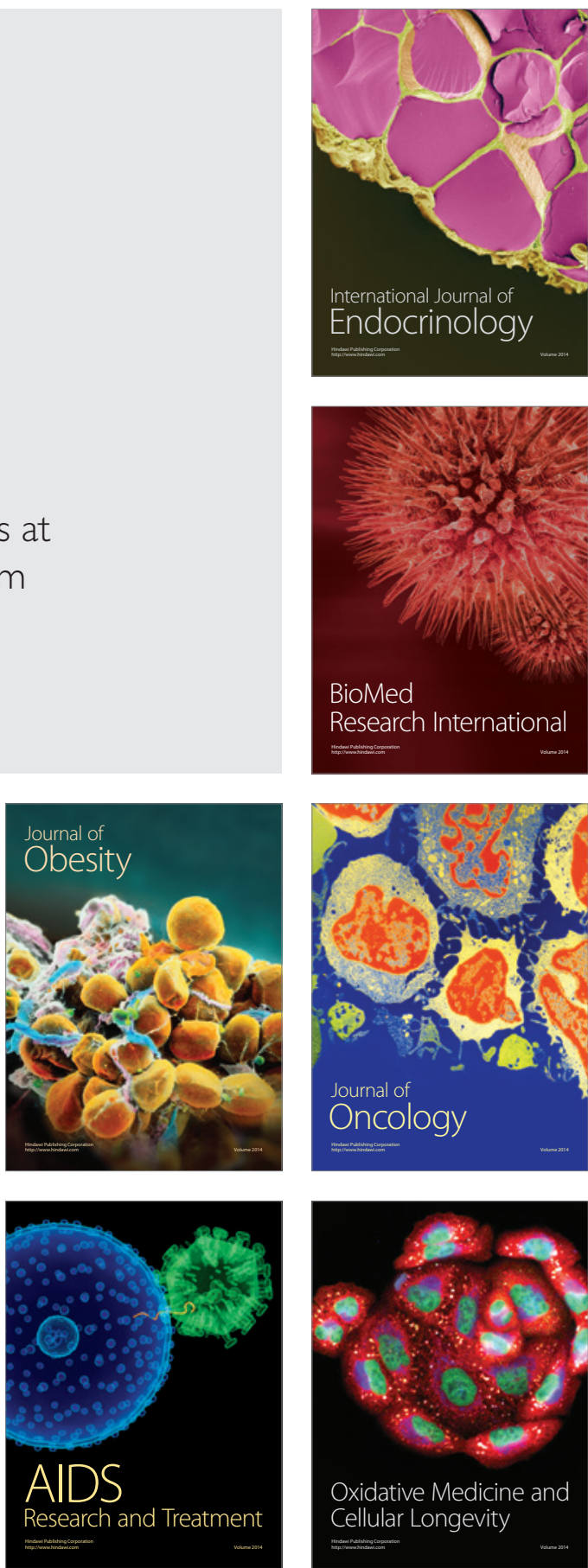